dimensional impurity channel (2DIC) on the bottom side of the quantum well. The peaks observed in the IQHE regime of the 2DEG reflect the resistivity of the 2DIC $\left(\rho_{x x}^{2 D I C}\right)$. In that regime, the coupling between the two channels leads to a nonequilibrium charge distribution in the 2DEG with very long RC characteristic times, which causes the peak phenomena to last for a long time. The influence of the 2DEG nonequilibrium charge distribution on the 2DIC resistivity pattern is observed as the hysteretic peaks. The density of 2 DIC, estimated at about $5.7 \times 10^{10} \mathrm{~cm}^{-2}$, can drop below the conducting limit when a negative bias is applied to the back-side gate, causing the hysteresis to disappear.

WIRAWAN PURWANTO

\section{Lattice-Dynamics Properties May Explain Low Dielectric Constant of Thin Films}

Soft-mode phonons are known to play a key role in the physics of ferroelectrics. The softening of one particular phonon usually results in a dramatic increase in the dielectric constant and an appearance of the ferroelectric phase. The relative variation of the soft-mode frequency with the dielectric constant is fully described by the Lyddane-Sachs-Teller (LST) formula.

Researchers from The Pennsylvania State University and Max Planck Institute for Solid State Research have reported in the March 23 issue of Nature the relationship between the lower dielectric constant of thin films and the reduced softening of the lowest optical-phonon mode. This research may explain the mechanism of low dielectric constant in thin films.

After applying the dead-layer modelwhich takes into account interface effects and results in a decrease of the average dielectric constant-to derive a temperature-dependent dielectric constant for thin $\mathrm{SrTiO}_{3}$ (STO) films, the researchers found that the model was not able to accurately describe the measured dielectric constants. According to the researchers, latticedynamics studies are necessary for a better explanation of the lower dielectric constant of thin films.

The researchers used pulsed laser deposition to prepare the samples: They heated the substrate to $720^{\circ} \mathrm{C}$ in an oxygen pressure of $100 \mathrm{mTorr}$, then cooled the as-deposited film to room temperature in 400 Torr of oxygen. They then deposited STO films with thicknesses of $0.5,1$, and $2 \mu \mathrm{m}$ on a $0.35-\mu \mathrm{m}$ conducting oxide $\mathrm{SrRuO}_{3}$ buffer layer.

Although the researchers observed optical-phonon peaks in STO films by means of conventional Raman scattering, the soft mode (the lowest optical mode) was not detected in this way. For this reason, they carried out far-infrared ellipsometry measurements at the National Synchrotron Light Source. Taking advantage of the high brightness of the synchrotron and Fourier transform infrared (FTIR) spectroscopy, the complex dielectric functions had been directly measured in the frequency range of $30-700 \mathrm{~cm}^{-1}$ and at a temperature range of $5-300 \mathrm{~K}$.

From the plots of the real and imaginary parts of the measured complex dielectric constants versus wave numbers, the soft-mode optical TO1 (transverse) phonon has been clearly observed, as well as other TO modes. It proved the feasibility of the experimental method.

In order to acquire the frequency of the soft mode, the researchers fit the experimental spectra to the dielectric function that takes into account the contribution of the phonon broadening. They found that the eigenfrequency of the soft mode (TO1) decreases as the temperature is lowered and saturates at $13 \mathrm{~cm}^{-1}$ instead of $62 \mathrm{~cm}^{-1}$ for the bulk crystal. On the other hand, similar eigenfrequencies of other longitudinal (LO) and TO modes have been observed both in the thin film and the bulk.

By comparing the results with the LST formula, the researchers observed the correlation between the harder soft mode in the thin film and a lower static dielectric constant. The LST relation between the optical-phonon eigenfrequencies and the static dielectric constant has been found to be fully maintained in thin films like $\mathrm{SrTiO}_{3}$ as well as in the bulk material. The researchers further said that "besides the dead-layer effect, the lower dielectric constant in STO thin films is due to their fundamental lattice dynamical propertiesspecifically, the soft-mode hardening."

In their discussion of the physical mechanisms for the soft-mode hardening, the researchers found that local strain and defects play key roles.

JIDONG HOU

\section{Chiral Diarylethene Dopant Induces Large Photostimulated Pitch Change in Nematic Liquid Crystals}

In an effort to explore photostimulated reversible phase changes in liquid crystals, researchers at Osaka Prefecture University, Kyushu University, and CREST have developed a new photoactive chiral chromophore-that is, a chiral cyclohexane containing two diarylethenes. In the April issue of Chemistry of Materials, the researchers refer to this compound as com- pound 1. They explored the photoisomerization of compound $\mathbf{1}$ and its influence on the properties of a doped nematic liquid crystal and observed a large and stable photostimulated pitch change in a chiral nematic phase induced by this compound.

With UV light irradiation (a mercury lamp, $254 \mathrm{~nm}, 10 \mathrm{~W}$ ), the researchers converted open-ring isomers of compound 1 to stable closed-ring isomers. Irradiated by visible light (a halogen lamp, $\lambda>480 \mathrm{~nm}$, $100 \mathrm{~W}$ ), the closed-ring isomers were switched back to open-ring isomers. The researchers doped a $1.0 \mathrm{wt} \%$ open-ring isomer of compound 1 into a nematic liquidcrystalline material, 4-cyano- $4^{\prime}$-pentylbiphenyl (K-15), at $26^{\circ} \mathrm{C}$ and achieved an apparent nematic phase. An induced cholesteric phase (chiral nematic phase) resulted at content higher than $5.0 \mathrm{wt} \%$. When the doped liquid crystal $(1.0 \mathrm{wt} \%)$ was irradiated with UV light for $3 \mathrm{~min}$, the original stable nematic texture turned into a cholesteric fingerprint pattern (observed by a polarization microscope) and the pitch of the liquid crystalline phase decreased from longer than $40 \mu \mathrm{m}$ to $11.8 \mu \mathrm{m}$ (measured by the droplet method). When irradiated with visible light for another $3 \mathrm{~min}$, the observed texture gradually returned to the stable nematic texture, and the measured pitch went back to longer than $40 \mu \mathrm{m}$. This switching cycle was performed more than 50 times without deterioration of the liquid-crystalline phase. The researchers said that the twisting power of the closedring isomers is much larger than that of the open-ring isomers and this accounts for the above photoresponsive behavior.

In addition, the researchers prepared an azobenzene derivative and compared its optical rotation value with that of compound 1 . They conclude that the large increase in the optical rotation value of compound 1 plays a role in the high efficiency of the phase change.

ZHENGMAO ZHU

\section{Intraband Electron Energy Relaxation in CdSe Quantum Dots is Dominated by Coulombic Coupling Between Electrons and Holes}

Femtosecond (fs) transient absorption measurements of CdSe quantum dots capped with either a ZnS layer or a pyradine layer indicate that electron intraband energy relaxation is dominated by Coulombic electron-hole coupling effects rather than by phonon-mediated processes (as is true of bulk materials) or Augerassisted processes (i. e., electron-hole recombination with the excess energy given to a hole). Researchers at Los 